\title{
Randomized transmission power for accelerated consensus in asymmetric WSNs
}

\author{
Silvana Silva Pereira and Alba Pagès-Zamora \\ Signal Processing and Communications Group (GPS) \\ Technical University of Catalonia (UPC), Barcelona - Spain \\ Email: silvana@gps.tsc.upc.edu, alba.pages@upc.edu
}

\begin{abstract}
The total energy consumed in a wireless sensor network until reaching a consensus is proportional to the product of the sum of the transmission power of each node and the convergence time. In a network where the nodes are allowed to transmit using different power levels, the minimization of this consumption is not trivial. In this paper, we propose a heuristic scheme of randomized power transmission such that the energy consumed by the network is balanced among the nodes and the convergence time is reduced with respect to a fixed symmetric topology that spends the same average transmission power. The proposed scheme establishes random links between nodes with different probabilities of connection, giving a random asymmetric topology at every iteration. Therefore, in order to minimize the convergence time of the algorithm, we use previous results on the analysis of the mean square error of the state.
\end{abstract}

\section{INTRODUCTION}

A sufficient condition to reach the average consensus in wireless sensor networks (WSNs) is that the topology of the underlying graph is undirected, leading to symmetric links between pairs of nodes at every time instant [1]. In other words, the instantaneous Laplacian matrix is symmetric and positive semidefinite. The assumption of a symmetric Laplacian at every time instant requires both the use of an acknowledgement procedure for the transmissions among the nodes and the control of the iteration time, so the simplicity of the average consensus algorithm is somehow broken. Most of the results found in literature for random topology networks assume however a symmetric Laplacian matrix, e.g. [2]-[4], and relate the convergence time of the average consensus algorithm to the second smallest eigenvalue of the average Laplacian. Few papers can be found assuming asymmetric topologies. In [5], the convergence to a common value is related to the second largest eigenvalue of the average weight matrix and necessary and sufficient conditions for almost sure convergence are given whenever the weight matrices fulfill certain conditions. Another example is [6] in which the authors prove the convergence to the average consensus also for asymmetric topologies but restricted to link weights tending to zero and therefore, at the cost of increasing the convergence

This work has been partially funded by the Spanish Science and Technology Commissions, the Catalan Government and FEDER funds under contracts TEC2006-06481/TCM, CONSOLIDER CSD2008-00010 COMONSENS and 2009SGR-01236. time. In our previous work in [7], we assume a random WSN where the communication links exist with nonzero probability and study the convergence of the algorithm in the mean square sense. A closed form expression for the mean square error (MSE) of the state is derived, along with the parameters that guarantee fastest convergence. This expression is derived under the assumptions of asymmetric instantaneous links but all with equal probability of connection, i.e. the Erdős-Rényi random graph model, which in practice is not realistic. The work in [8] generalizes the analysis of [7] to the case of different probabilities of connection, and derives an upper bound for the MSE of the state along with an approach to determine the link weight that minimizes the convergence time.

The energy consumption in a WSN until reaching a consensus is proportional to the product of the sum of the transmission power of each node and the convergence time. After reaching a consensus, we assume that the nodes switch to a save-energy mode in order to extend the network lifetime. In [9], for instance, the authors assume that all the nodes transmit at the same power level and find out that the energy consumption of a randomly uniform deployed WSN is similar to a WSN deployed on a uniform grid as the number of nodes increases. However, it is not difficult to check that the energy consumption is minimum when the network is almost fully connected. The generalization of this minimization problem to the situation in which nodes may transmit at different power levels is not trivial. Actually, it is a non-convex problem as opposed to the minimization problems in [10] where only the convergence time is taken into account. In this paper, we focus on the convergence time and the energy consumption of the consensus algorithm in WSNs with asymmetric random topologies, which was not treated in detail in [8]. We propose a heuristic although practical scheme of power transmission in WSNs such that the amount of energy consumed by the network when running the consensus algorithm is balanced among the nodes and lower than in the case of the fixed symmetric topology. The proposed scheme leads to a nonsymmetric topology and establish links between nodes with different probabilities. Therefore, the results obtained in [8] for the MSE are useful to better understand the performance obtained with the proposed scheme. The paper is organized as 
follows. In Section II we introduce some useful definitions of graph theory for random graphs and in Section III we present the consensus algorithm with the proposed randomized transmission power scheme. In Section IV we analyze the MSE for the model and the convergence of its upper bound. Simulation results and conclusions are included in Section V and VI respectively.

\section{GRAPH THEORY CONCEPTS}

The information flow among the nodes of a network with a random topology can be described by a directed graph $\mathcal{G}(k)=$ $\{\mathcal{V}, \mathcal{E}(k)\}$, where $\mathcal{E}(k)$ is the set of edges $e_{i j}$ at time $k, \forall$ $i, j=\{1, \cdots, N\}$, such that the information flows from node $j$ to node $i$, and $\mathcal{V}$ is the constant set of nodes [11]. We assume that $\mathcal{G}(k)$ has no loops or multiple edges, i.e. $e_{i i}=0 \forall k$. The set of neighbors of node $i$ is denoted $\mathcal{N}_{i}(k)=\left\{j \in \mathcal{V}: e_{i j} \in\right.$ $\mathcal{E}(k)\}$, and represents the set of nodes sending information to node $i$ at time $k$. The instantaneous adjacency matrix of $\mathcal{G}(k)$, denoted $\mathbf{A}(k) \in \mathbb{R}^{N \times N}$, is random and has entries equal to 1 if $e_{i j} \in \mathcal{E}(k)$ and 0 otherwise. The degree matrix $\mathbf{D}(k) \in \mathbb{R}^{N \times N}$ is a diagonal matrix whose entries are given by $[\mathbf{D}(k)]_{i i}=$ $\sum_{j=1}^{N}[\mathbf{A}(k)]_{i j}$. The instantaneous Laplacian of the graph is a random matrix defined as $\mathbf{L}(k)=\mathbf{D}(k)-\mathbf{A}(k)$, with smallest eigenvalue equal to zero for all $k$ [12].

\section{CONSENSUS WITH RANDOMIZED POWER}

Consider a WSN composed of $N$ nodes indexed with $i=$ $\{1, \cdots, N\}$ and a scalar value $x_{i}(k)$ defined as the state of node $i$ at time $k$. The state is initialized at each node at $k=0$ with the value of a single measurement and evolves in time according to the following difference equation [1]

$$
x_{i}(k)=x_{i}(k-1)+\epsilon \sum_{j \in \mathcal{N}_{i}(k)}\left(x_{j}(k-1)-x_{i}(k-1)\right),
$$

for $k>0$, where the link weight $\epsilon$ is a positive constant chosen to satisfy convergence conditions and $\mathcal{N}_{i}(k)$ is the set of neighbors of node $i$ at time $k$. We propose a scheme where at each iteration the nodes transmit using a power level randomly selected from a predefined set of values. Therefore, the transmission power at each node varies with time and is, in general, different among nodes at the same time instant. These power levels describe different concentric circles of connectivity, centered at the transmitting node, and with a radius denoted $\rho_{i}(k)$ for node $i$ at time $k$, which is proportional to the square root of the associated transmit power level. Without loss of generality, we define the set containing all the possible radii arranged in increasing order as

$$
R=\left\{\rho^{(1)}, \rho^{(2)}, \cdots, \rho^{(|R|)}\right\},
$$

so that $\rho_{i}(k) \in R$, where $\rho^{(1)}$ is the connectivity radius associated with the minimum power level, $\rho^{(|R|)}$ is the connectivity radius associated with the maximum power level, and |.| denotes the cardinality of a set. For instance, if at iteration $k$ the distance $d(i, j)$ between two nodes $\{i, j\} \in \mathcal{V}$ satisfies $d(i, j)<\rho_{i}(k)$, then node $j$ receives the information from node $i$ at time $k$. Thus, the set of neighbors for every node and consequently the adjacency matrix $\mathbf{A}(k)$, varies randomly from iteration to iteration depending on the instantaneous choice of power levels. For simplicity, we assume that the power levels in (2) have all the same probability equal to $\pi=\frac{1}{|R|}$. Then, the nodes lying inside the first circle centered at node $i$ will receive the information from node $i$ with probability 1 , while the nodes lying only in the last annulus receive the information from node $i$ with probability $\pi$. The nodes outside the outer circle receive information with probability 0 .

Let $\mathbf{P}$ denote the connection probability matrix, whose entry $[\mathbf{P}]_{i j}$ is the probability that a link between two nodes $\{i, j\}$ exists for a given deployment, with $[\mathbf{P}]_{i j}=[\mathbf{P}]_{j i}$. Applying the randomized power scheme described above and letting $\rho^{0}=0$, the entries of $\mathbf{P}$ are given by

$$
[\mathbf{P}]_{i j}=\left\{\begin{array}{cc}
(|R|-l+1) \pi & \text { if } \rho^{(l-1)}<d(i, j)<=\rho^{(l)} \\
0 & \text { otherwise }
\end{array}\right.
$$

for all $\{i, j\} \in \mathcal{V}$ and $l \in\{1, \cdots,|R|\}$. The matrix $\mathbf{A}(k)$ for a network with these transmission characteristics is nonsymmetric random and satisfies $\overline{\mathbf{A}}=\mathbf{P}$, where the bar denotes expected value. The matrix $\mathbf{P}$ and its properties will be used in the convergence analysis, as we will see in Section IV.

\section{A. Asymmetric random links}

Let $\mathbf{x}(k) \in \mathbb{R}^{N \times 1}$ denote the vector containing all the states of the network at time $k>0$. Considering the communication scheme in (1) with instantaneous connectivity radii $\rho_{i}(k) \in R$ for each node, the evolution of $\mathbf{x}(k)$ can be written in matrix form as follows

$$
\mathbf{x}(k)=\mathbf{W}(k-1) \mathbf{x}(k-1)
$$

where the weight matrix can be expressed as

$$
\mathbf{W}(k)=\mathbf{I}-\epsilon \mathbf{L}(k)
$$

$\mathbf{I} \in \mathbb{R}^{N \times N}$ is the identity matrix, $\mathbf{L}(k)$ is the instantaneous Laplacian and $\epsilon$ is equal for all the iterations. Due to the random nature of $\mathbf{A}(k), \mathbf{L}(k)$ is random and in general nonsymmetric with mean $\overline{\mathbf{L}}=\overline{\mathbf{D}}-\mathbf{P}$. Note that he entries of $\mathbf{L}(k)$ are however not independent among each other. Therefore, the matrices $\{\mathbf{W}(k), \forall k \geq 0\}$ are by construction random, independent of each other, non-symmetric and row-stochastic with one eigenvalue equal to one and associated right eigenvector $\mathbf{1} \in \mathbb{R}^{N \times 1}$ (all-ones vector), and satisfy $\overline{\mathbf{W}}=\mathbf{I}-\epsilon \overline{\mathbf{L}}$. The dynamical range of $\epsilon$ guaranteeing mean square convergence assuming a symmetric $\mathbf{L}(k)$ is derived in [4], whereas the dynamical range of $\epsilon$ for asymmetric $\mathbf{L}(k)$, along with the optimum $\epsilon$ guaranteeing fastest mean square convergence of the algorithm are derived in [7] assuming equal probabilities of connection. The dynamical range of $\epsilon$ guaranteeing almost sure convergence can be derived from [5] for nonnegative matrices $\{\mathbf{W}(k), \forall k\}$. At this point, we aim at determining the value of $\epsilon$ that provides a fast convergence of the consensus algorithm in (3), for weight matrices that are not necessarily nonnegative. For that purpose, we use below the theoretical results derived in [8] with regards to the upper bound of the MSE of the state. 


\section{MSE ANALYSIS}

Let $\mathbf{x}(0)=\left[\begin{array}{llll}x_{1}(0) & x_{2}(0) & \cdots & x_{N}(0)\end{array}\right]^{T}$ be the vector of measurements taken by the sensors, modeled as independent identically distributed (i.i.d.) random variables with mean $x_{m}$ and variance $\sigma_{0}^{2}$. Due to the random nature of both $\mathbf{x}(0)$ and the matrices $\{\mathbf{W}(k), \forall k\}$ in (4), we consider the convergence of $\mathbf{x}(k)$ to the mean average consensus, defined as

$$
\mathbf{x}_{m a}=\frac{1}{N} \mathbf{1}^{T} \mathbb{E}[\mathbf{x}(0)] \mathbf{1}=x_{m} \mathbf{1}
$$

where $\mathbb{E}[$.$] denotes expected value. Our aim is to minimize$ the overall energy consumption of the network; therefore, we aim at finding the $\epsilon$ that minimizes the convergence time of $\mathbf{x}(k)$ in (3). We relate the convergence of the algorithm to the convergence of the MSE of the state, defined as

$$
\operatorname{MSE}(x(k))=\frac{1}{N} \mathbb{E}\left[\left\|\mathbf{x}(k)-\mathbf{x}_{m a}\right\|_{2}^{2}\right] .
$$

Because of the lack of a closed form expression for the $\operatorname{MSE}(x(k))$ above, we focus on the upper bound derived in [8], which can be used to characterize the convergence time of the algorithm under different probabilities of connection for the links. For convenience, let us define the matrix

$$
\mathbf{R}_{\mathbf{w}}(k)=\mathbb{E}\left[\left(\prod_{l=1}^{k} \mathbf{W}(k-l)\right)^{T}\left(\prod_{l=1}^{k} \mathbf{W}(k-l)\right)\right] .
$$

We can now state our main proposition:

Proposition 1: Consider the iterative algorithm in (3) with $N$ nodes, i.i.d. initial values $\mathbf{x}(0)$ with mean $x_{m}$, variance $\sigma_{0}^{2}$, and randomized transmission power with connectivity radii as defined in (2). Assuming that $\mathbf{R}_{\mathbf{w}}(k)$ in (6) has largest eigenvalue one for all $k$, the empirical $\operatorname{MSE}(x(k))$ in (5) is upper bounded by

$$
\operatorname{MSE}(x(k)) \leq \frac{\sigma_{0}^{2}}{N}\left(N+\left(\operatorname{tr}\left(\mathbf{C}_{\mathbf{w}}\right)-N\right) \frac{1-\lambda_{N}^{k-1}\left(\mathbf{C}_{\mathbf{w}}\right)}{1-\lambda_{N}\left(\mathbf{C}_{\mathbf{w}}\right)}\right)
$$

where $\mathbf{C}_{\mathbf{w}}=\mathbb{E}\left[\mathbf{W}(k) \mathbf{W}^{T}(k)\right]$ for all $k$, and $\lambda_{N}\left(\mathbf{C}_{\mathbf{w}}\right)$ denotes its smallest eigenvalue.

Proof: The proof follows directly from Theorem 3 in [8] and is omitted here due to lack of space.

Note that the upper bound in (7) converges if and only if $\left|\lambda_{N}\left(\mathbf{C}_{\mathbf{w}}\right)\right|<1$, and the convergence time of the upper bound decreases as $\left|\lambda_{N}\left(\mathbf{C}_{\mathbf{w}}\right)\right| \rightarrow 0$. After some matrix manipulations, the matrix $\mathbf{C}_{\mathbf{w}}$ can be rewritten as follows

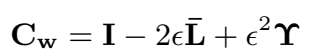

where the matrix $\boldsymbol{\Upsilon}=\mathbb{E}\left[\mathbf{L}(k) \mathbf{L}^{T}(k)\right]$ has entries

$$
\begin{aligned}
& {[\mathbf{\Upsilon}]_{i i}=2 \sum_{l=1}^{N}[\mathbf{P}]_{i l}+\sum_{l=1}^{N} \sum_{\substack{m=1 \\
m \neq l}}^{N}[\mathbf{P}]_{i l}[\mathbf{P}]_{i m},} \\
& {[\mathbf{\Upsilon}]_{i j}=-[\mathbf{P}]_{i j}\left(\sum_{l=1}^{N}[\mathbf{P}]_{i l}+\sum_{l=1}^{N}[\mathbf{P}]_{j l}\right)+\sum_{\substack{l=1 \\
l \neq j}}^{N} \Phi_{l}, i \neq j}
\end{aligned}
$$

with $\Phi_{l}=[\mathbf{P}]_{i l}$ if $\rho^{(|R|)}>d(i, l)>d(j, l)$ and $\Phi_{l}=[\mathbf{P}]_{j l}$ if $\rho^{(|R|)}>d(j, l) \geq d(i, l)$ for all $l$. Since the matrix $\mathbf{C}_{\mathbf{w}}$ in (8) depends on the matrix $\mathbf{P}$, it can be computed off-line whenever we have knowledge of the probabilities of connection. For our randomized transmission power model, the matrix $\mathbf{P}$ can be derived when the node locations (which we assume fixed) and the set of power levels $R$ in (2) are known. Then, using an exhaustive search over all values of $\epsilon$ in a given interval we can choose the $\epsilon$ that minimizes $\left|\lambda_{N}\left(\mathbf{C}_{\mathbf{w}}\right)\right|$.

\section{A. Evaluation parameters}

In Section $\mathrm{V}$ we evaluate the performance of a network implementing the consensus algorithm using the proposed transmission power scheme in terms of convergence time and energy consumption. We also compare it with two networks with fixed symmetric topology where the nodes transmit using constant transmission power. In the first one, the transmission power is equal to the average power of the randomized network, such that the connectivity radius for each node is given by

$$
\rho_{\text {ave }}=\sqrt{\frac{1}{|R|} \sum_{l=1}^{|R|}\left(\rho^{(l)}\right)^{2}} .
$$

This network will spend the same transmission power as the randomized power network on average over time. In the second one, the transmission power is equal to the maximum power of the randomized network, such that the connectivity radius for each node is equal to $\rho_{\max }=\rho^{(|R|)}$. Note that this case is interesting because, as mentioned in the introduction, the minimum energy consumption of a network implementing the consensus algorithm with all the nodes transmitting at the same power is attained when the network is almost fully connected. Due to the dimensions of the network we simulate and the power levels we apply, we are close to that situation when all the nodes transmit using the maximum power available. As it will be shown, the overall energy consumed until reaching a consensus will be higher in these networks than in the randomized one.

\section{Simulation RESUltS}

We simulate a WSN composed of $N=100$ nodes uniformly deployed in a squared area of dimensions $100 \times 100$, where each node measurement is modeled as an independent Gaussian random variable with mean $x_{m}=-4$ and variance $\sigma_{0}^{2}=25$. A total of 10 thousand realizations of $\mathbf{x}(0)$ were simulated to obtain the empirical $\operatorname{MSE}(x(k))$ in (5). We evaluate the performance of the randomized power network with several power levels and applying constant link weights equal to $\epsilon^{*}$, where $\epsilon^{*}$ is the value that minimizes the convergence time of the upper bound in (7). The instantaneous radius of connectivity for each node, denoted hereafter as $\rho_{\text {var }}$, takes one of the values $R=\{5,10,15,30\}$. The fixed topology networks use constant transmission power and $\epsilon_{\text {opt }}=\frac{2}{\lambda_{2}(\mathbf{L})+\lambda_{N}(\mathbf{L})}[10]$; the first one has transmission power equal to the average power of the randomized one, with connectivity radius defined in (9) and equal to $\rho_{\text {ave }} \simeq 17.67$. The second one uses 
a transmission power equal to the maximum power of the randomized network, i.e. $\rho_{\max }=30$.

Figure 1 shows the empirical $\operatorname{MSE}(x(k))$ plotted in $\mathrm{dB}$ as a function of the iteration index for the three cases. The curve for the fixed network with radius $\rho_{\text {ave }}$ is shown in dashed line, whereas the network with radius $\rho_{\max }$ is depicted with line-dots. The curve for the randomized power network is shown in dashed-dotted line and the benchmark value $\frac{\sigma_{0}^{2}}{N}$ is included in solid line. As expected, the curves for the fixed topology networks reach the benchmark, since $\mathbf{L}(k)$ is in both cases time-invariant and symmetric. First, we observe that the curve for the randomized power network converges faster than the curve for the average power case (ca. 84 and 164 iterations respectively), although it stays over due to the nonsymmetry of $\mathbf{L}(k)$. This is due to the fact that at some time instants, a given node $i$ can communicate with a node $j$ far away (with maximum distance $d(i, j)<\rho^{(|R|)}$ ), increasing the instantaneous connectivity of the network. These results show that using the randomized power scheme, the convergence time of the consensus algorithm can be improved at the same overall power consumption. This means that the overall energy consumption to reach consensus is lowered, although at the cost of a reduction in the accuracy of the estimation. In addition, the value of $\epsilon^{*}$ that minimizes the convergence of the upper bound seems to be a good choice to reduce the convergence time of the empirical $\operatorname{MSE}(x(k))$ as well. If we compare the network using maximum transmission power to the randomized power one, the former converges faster, i.e. 40 vs 84 iterations. However, the overall energy consumption in the fixed network is greater than in the randomized power one, since the energy spent by every node is proportional to $K \times \rho_{\max }^{2}$, where $K$ denotes the convergence time. The results for this and for other combinations of power levels are included in TABLE I, showing the approximate number of iterations to reach consensus and the consumed power by each node until reaching consensus in terms of $K \times$ radius $^{2}$. Despite numerical differences, in all cases the randomized power network converges faster than the fixed network with average transmission power.

\section{CONCLUDING REMARKS}

In this paper, we propose a simple power transmission scheme that extends the lifetime of WSNs running consensus algorithms. The nodes transmit using different power levels at every time instant, selected independently of other nodes and with equal probability. The convergence time is reduced with respect to a fixed topology where the nodes transmit using the same average power; therefore, the total energy required to reach a consensus is reduced and the network lifetime is lengthened. The randomized transmission power scheme is also more energy efficient than a fully connected network. The price to be paid for significantly reducing the energy consumption is a reduction in the accuracy of the estimation. The preference of improving either the accuracy in the estimation or the energy consumption can be determined by the final application.

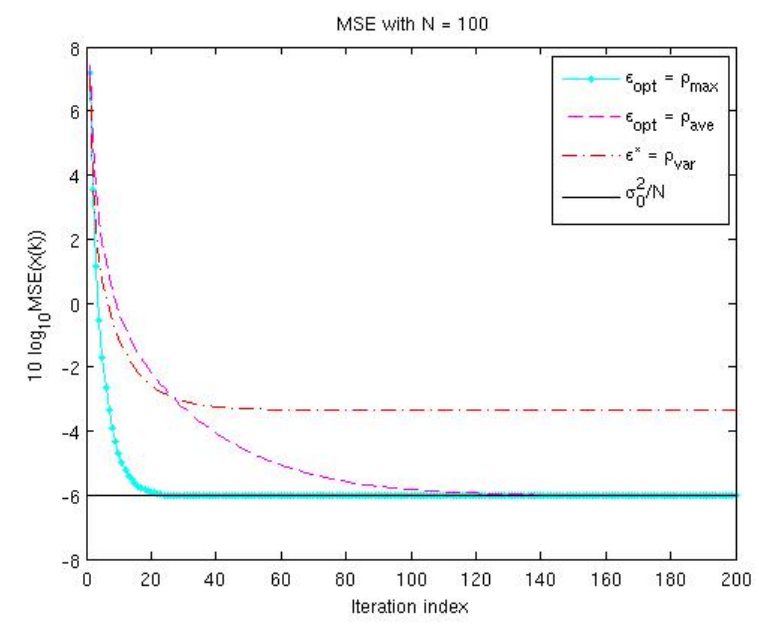

Fig. 1. Empirical $\operatorname{MSE}(x(k))$ in $\mathrm{dB}$ as a function of $k$ for a randomized power network with variable radius of connectivity $\rho_{v a r}$, and for fixed topology networks with fixed power and radii $\rho_{\text {ave }} \simeq 17.67$ and $\rho_{\max }=30$.

TABLE I

SIMULATION RESULTS FOR SEVERAL POWER COMBINATIONS.

\begin{tabular}{|c|r|r|r|r|r|r|}
\hline Connectivity radii & \multicolumn{3}{|c|}{ No. of Iterations $(K)$ with: } & \multicolumn{3}{|c|}{ Energy consumption $\propto$} \\
\cline { 2 - 7 }$R \&\left(\rho_{\text {ave }}\right)$ & $\rho_{\max }$ & $\rho_{\text {var }}$ & $\rho_{\text {ave }}$ & $K \cdot \rho_{\max }^{2}$ & $K \cdot \rho_{\text {var }}^{2}$ & $K \cdot \rho_{\text {ave }}^{2}$ \\
\hline$\{5,10,15,30\}(17.7)$ & 40 & 84 & 164 & 36000 & 26250 & 51250 \\
\hline$\{5,10,15,50\}(26.7)$ & 16 & 25 & 73 & 40000 & 17812 & 52012 \\
\hline$\{10,20,30,60\}(35.3)$ & 12 & 22 & 29 & 43200 & 27500 & 36250 \\
\hline$\{10,20,70\}(42.4)$ & 9 & 13 & 21 & 44100 & 23400 & 37800 \\
\hline
\end{tabular}

\section{REFERENCES}

[1] R. Olfati-Saber and R. Murray, "Consensus problems in networks of agents with switching topology and time-delays," IEEE Transactions on Automatic Control, vol. 49, no. 9, pp. 1520-1533, Sept. 2004.

[2] Y. Hatano and M. Mesbahi, "Agreement over random networks," in Proc. 43rd IEEE Conference on Decision and Control, vol. 2, 14-17 Dec. 2004, pp. 2010-2015Vol.2.

[3] S. Kar and J. Moura, "Distributed average consensus in sensor networks with random link failures," in Proc. IEEE ICASSP, vol. 2, 15-20 April 2007, pp. II-1013-II-1016.

[4] - "Sensor networks with random links: Topology design for distributed consensus," IEEE Transactions on Signal Processing, vol. 56 , no. 7, pp. 3315-3326, 2008.

[5] A. Tahbaz-Salehi and A. Jadbabaie, "A necessary and sufficient condition for consensus over random networks," IEEE Transactions on Automatic Control, vol. 53, no. 3, pp. 791-795, 2008.

[6] M. G. Rabbat, R. D. Nowak, and J. A. Bucklew, "Generalized consensus computation in networked systems with erasure links," in Proc. IEEE SPAWC, 2005, pp. 1088-1092.

[7] S. Silva Pereira and A. Pages-Zamora, "Fast mean square convergence of consensus algorithms in WSNs with random topologies," in Proc. IEEE ICASSP, 2009, pp. 2213-2216.

[8] — , "Mean square convergence of consensus algorithms in random WSNs," submitted to IEEE Transactions on Signal Processing.

[9] S. Barbarossa, G. Scutari, and A. Swami, "Achieving consensus in selforganizing wireless sensor networks: The impact of network topology on energy consumption," in Proc. IEEE ICASSP, vol. 2, 2007, pp. II841-II-844.

[10] L. Xiao and S. Boyd, "Fast linear iterations for distributed averaging," in Proc. 42nd IEEE Conference on Decision and Control, vol. 5, 9-12 Dec. 2003, pp. 4997-5002.

[11] C. Godsil and G. Royle, Algebraic graph theory. Graduate Texts in Mathematics. Berlin, Germany: Springer-Verlag, 2001, vol. 207.

[12] R. Horn and C. Johnson, Matrix analysis. Cambridge University Press, 2006. 